\title{
Zwangsmaßnahmen im psychiatrischen Alltag - und die Kultur der (wissenschaftlichen) Beschäftigung damit
}

\author{
The Clinical Practice of Coercive Measures in Psychiatric Hospital Care - \\ and the Culture of Dealing (Scientifically) with this Topic
}

Autor

Institute

\author{
Thomas W. Kallert ${ }^{1,2}$ \\ 1 Klinik und Poliklinik für Psychiatrie und Psychotherapie, Universitätsklinikum Carl Gustav Carus \\ an der Technischen Universität Dresden \\ 2 Klinik für Psychiatrie, Psychosomatik und Psychotherapie, Parkkrankenhaus Leipzig-Südost
}

Bibliografie

DoI $10.1055 / \mathrm{s}-2006-952001$

Psychiat Prax 2007; 34,

Supplement 2: S179-S180

(c) Georg Thieme Verlag KG

Stuttgart · New York .

ISSN 1611-8332

Korrespondenzadresse

Prof. Dr. med. habil.

Thomas W. Kallert

Park-Krankenhaus Leipzig-

Südost $\mathrm{GmbH}$, Klinik für

Psychiatrie, Psychosomatik

und Psychotherapie

Morawitzstraße 2

04289 Leipzig

Thomas.Kallert@mailbox.

tu-dresden.de
Liebe Leserin, lieber Leser,

obwohl klinisch unverändert keine Seltenheit $[1,2]$ und historisch gesehen lange Bestandteil psychiatrischen Handelns [3], ist der Kenntnisstand über verschiedene Aspekte der Anwendung von Zwangsmaßnahmen unzureichend. Dies kontrastiert zu der Intensität der verständlichen Kritik hieran, die von betroffenen PatientInnen und von verschiedenen, der Respektierung von Menschenrechten verpflichteten Organisationen geübt wird [4].

Die gravierenden Mängel beziehen sich dabei auf basale Bereiche üblicher medizinischer Wissensaufbereitung. Bezogen auf die Häufigkeit der Anwendung verschiedener Maßnahmen liegen auf nationaler Ebene z.B. für Deutschland strittig diskutierte Zahlen nur für Zwangseinweisungen, und kaum für weitere Zwangsmaßnahmen, vor [1,5 - 7], deren Validität unter z. T. nicht eindeutigen Definitionen in verschiedenen Datensammlungen und fehlenden Dokumentationsstandards leidet. Bis dato hat die psychiatrische Versorgungsforschung auch nur wenige Befunde dazu vorgelegt, ob und wie die Häufigkeit der Anwendung von Zwangsmaßnahmen als Qualitätsindikator psychiatrischer Versorgung dienen kann [8]. Zu Indikationen existiert lediglich ein Konsens auf dem Niveau von Expertenmeinungen, was die geringe Datenbasis an Leitlinien verdeutlicht, die auf hohem Evidenzniveau lediglich zu praktischen Aspekten der Zwangsmedikation $[9,10]$ Aussagen treffen. Zur Effektivität von Zwangsmaßnahmen hinsichtlich verschiedener Outcomedomänen können kaum gesicherte differenzielle Aussagen gemacht werden; gleiches gilt für die Anwendung alternativer Maßnahmen [11]. Zu den wichtigen Bereichen potenziell wirksamer Programme zur Reduktion von Zwangsmaßnahmen [12,13] sowie zur Prävention derselben im klinischen Alltag [14] gibt es im deutschen Sprachraum noch keine überzeugenden Befunde. National verbindliche Leitlinien auf heutzutage gefordertem Qualitätsniveau sind für die Anwendung verschiedener Zwangsmaßnahmen nicht verfügbar. Zentrale rechtspraktische Fragen harren zumindest einer transnationalen Harmonisierung [15], aber auch potenzielle Unterschiede in der Rechtspraxis zwischen den einzelnen Bundesländern benötigen eine vertiefte systematische Erforschung. Zwangskonzepte, die insbesondere das subjektive Zwangserleben von PatientInnen einbeziehen, müssen weiter inhaltlich ausdifferenziert und in ihrem klinischen Stellenwert exploriert werden.

Diese Mängel in der wissenschaftlichen Bearbeitung tragen vermutlich entscheidend dazu bei, dass neueste Auflagen großer deutscher psychiatrischer Therapielehrbücher [16] sowie Neufassungen von Weiterbildungsordnungen im Gebiet Psychiatrie und Psychotherapie [17] diese Thematik nicht adressieren - eine Praxis, die künftig dringend revisionsbedürftig ist.

All dies veranlasst, neue Übersichten $\mathrm{zu}$ mit Zwangsmaßnahmen assoziierten Einstellungsund ethischen Aspekten, aktuelle empirische praxisorientierte Ergebnisse zu dem Thema sowie Ausschnitte der Bearbeitung von Rechtsfragen und klinischen Praxisempfehlungen in einem Sonderheft der Zeitschrift „Psychiatrische Praxis“ zusammenzustellen. Dieses geht auf ein Symposion zurück, das am 17.2.2006 im Universitätsklinikum an der TU Dresden stattgefunden hat.

Im Einzelnen beschäftigen sich die Artikel des Sonderheftes mit folgenden Inhaltsbereichen. C. Lauber und W. Rössler fassen die Sichtweise der öffentlichen Meinung und der Professionellen in der Psychiatrie auf Zwangsaufnahmen in das psychiatrische Krankenhaus zusammen. T. Steinert demonstriert am Beispiel der zwangsweisen Unterbringung und Behandlung schizophren Erkrankter nicht nur das ethische Spannungsfeld von Patientenautonomie, Durchführung wirksamer Behandlung und Schadensvermeidung. Vielmehr legt er auch Befunde vor, die Einflussvari- 
ablen aller Beteiligten auf ethische Haltungen, wie z. B. professionelle Stellung, Nationalität und eigene Erfahrungen mit psychisch Erkrankten, verdeutlichen. A. Spengler reflektiert die rechtlichen, administrativen und methodischen Problemstellungen, die eine Interpretation versorgungsepidemiologischer Daten erschweren. Vor diesem Hintergrund präsentiert er zudem nationale Daten zur Entwicklung von Zwangseinweisungen über einen mehr als 10-jährigen Zeitraum, die einen v.a. auf betreuungsrechtliche Verfahren zurückzuführenden Anstieg erkennen lassen. H. J. Salize et al. belegen, dass sich auf der gegenwärtigen Datenbasis (Geschäftsberichte der Amtsgerichte) ein um den Pflegeheimanteil bereinigtes Schätzmodell für Zahl und zeitliche Trends unfreiwilliger Maßnahmen beschreiben und auf Bundesländerebene differenzieren lässt. Zentrale Ergebnisse dieser Modellbildung sind, dass im Zeitraum 1993 bis 2003 deutschlandweit der Anteil der Unterbringungen an den jährlichen Aufnahmen in die Allgemeinpsychiatrie weitgehend gleich bleibt, der um den Pflegeheimanteil bereinigte Anteil unterbringungsähnlicher Maßnahmen jedoch ansteigt. Letztgenannter Befund ist für die klinische Versorgungsrealität irritierend und dringend erklärungsbedürftig.

Die von G. Längle und W. Bayer berichteten Befragungsergebnisse von zwei richterlich im psychiatrischen Krankenhaus untergebrachten Patientengruppen zeigen, dass diese Maßnahme sowohl im direkten zeitlichen Kontext als auch mit langem Abstand mehrheitlich als ungerechtfertigt empfunden wird. Trotz zunehmender Behandlungseinsicht werden diesbezüglich eher Extrempositionen vertreten. R. Ketelsen et al. stellen dar, wie eine kooperative Zusammenarbeit von Kliniken mit dem Ziel der Qualitätssicherung und Reduktion von Zwangsmaßnahmen in einem mehrjährigen Zeitraum zu diesbezüglich relevanten Veränderungen im klinischen Alltag führt. V. Martin et al. zeigen anhand von Daten eines erstmals durchgeführten länderübergreifenden Klinikvergleichs, dass in Deutschland und der Schweiz unterschiedliche nationale Muster in der Anwendung von Isolierungen und Fixierungen bestehen.

B. Gericke und T. Kallert legen Befunde einer großen Klientel von abhängigkeitskranken Maßregelvollzugspatienten vor. Im Zentrum der Untersuchung steht die Identifizierung von prädiktiven Markern für eine Aussichtslosigkeit dieser Maßnahme. Spezifische Sozialisationserfahrungen, geringe soziale Integration vor der Unterbringung sowie Entweichungen aus dieser imponieren als prognostisch ungünstige Faktoren, die für eine breite Interpretation Raum bieten.

G. Hegendörfer stellt bisherige Legislativvorhaben in Deutschland zum kontrovers diskutierten Thema der ambulanten Zwangsbehandlung Beispielen gesetzlicher Regelungen außerhalb Deutschlands entgegen, zeigt auf, dass die Einführung der ambulanten Zwangsbehandlung sowohl auf Bundesebene i.R. des zivilrechtlichen Betreuungsrechtes als auch auf Landesebene im Bereich des öffentlich-rechtlichen Unterbringungsrechts letztlich gescheitert ist, und benennt wesentliche rechtliche Kritikpunkte an der ambulanten Zwangsbehandlung.

T. Kallert et al. berichten über eine erstmalig im europäischen Kontext erarbeitete Empfehlung zur Durchführungspraxis von Fixierungen, die als Beitrag zur transnationalen Harmonisierung diesbezüglicher klinischer Praxis verstanden werden kann: rechtliche und klinische Vorbedingungen, berufsgruppenspezifische Handlungsanweisungen und praxisrelevante prozedurale Aspekte werden adressiert.

Es bleibt zu hoffen, dass diesem Heft viele weitere Initiativen zur Darstellung wissenschaftlicher Befunde auf dem Gebiet der An- wendung von Zwangsmaßnahmen im psychiatrischen Alltag folgen werden. Die vielfältigen praktischen Bemühungen um Reduktion der Anwendung von Zwangsmaßnahmen verdienen eine fundierte empirische Unterlegung. Nur so kann sich eine Kultur der (wissenschaftlichen) Beschäftigung mit Zwangsmaßnahmen entwickeln.

\section{Danksagung}

Ohne die Unterstützung der Firmen Janssen-Cilag, Lilly Deutschland, Novartis Pharma, Merz Pharmaceuticals und Pfizer Pharma wäre die Erstellung dieses Sonderheftes nicht möglich gewesen.

\section{Literatur}

1 Salize HJ, Spengler A, Dressing $H$. Zwangseinweisungen psychisch Kranker - wie spezifisch sind die Unterschiede in den Bundesländern? Psychiat Prax 2007; 34 (Sonderheft 2): S196-S202

2 Martin V, Bernhardsgrütter R, Göbel R, Steinert T, Arbeitskreis zur Prävention von Gewalt und Zwang in der Psychiatrie, Qualitätszirkel Benchmarking Zwangsmaßnahmen. Ein Vergleich von Schweizer und deutschen Kliniken in Bezug auf die Anwendung von Fixierung und Isolierung. Psychiat Prax 2007; 34 (Sonderheft 2): S212 - S217

3 Saussure R de. Philippe Pinel. In: Kolle K (Hrsg): Große Nervenärzte. Band 1. 2. Aufl. Stuttgart, New York: Thieme Verlag, 1970: 216-235

4 Working Party on Psychiatry and Human Rights. "White paper" on the protection of the human rights and dignity of people suffering from mental disorder, especially those placed as involuntary patients in a psychiatric establishment. Brussels: Committee of Ministers of the Council of Europe, 2000

5 Spengler A, Dressing H, Koller M, Salize HJ. Zwangseinweisungen - bundesweite Basisdaten und Trends. Nervenarzt 2005; 76: 363-370

6 Müller P, Dressing H, Salize HJ. Pro und Kontra: Zunahme von Zwangseinweisungen psychisch Kranker. Psychiat Prax 2006; 33: 157-159

7 Kallert T. Nehmen Zwangseinweisungen in Deutschland wirklich zu? Die Psychiatrie 2005; 2: 231 - 243

8 Martin V, Kuster W, Baur M, Bohnet U, Hermelink G, Knopp M, Kronstorfer M, Martinez-Funk B, Roser M, Voigtländer W, Brandecker W, Steinert $T$. Die Inzidenz von Zwangsmaßnahmen als Qualitätsindikator in psychiatrischen Kliniken. Probleme der Datenerfassung und -verarbeitung und erste Ergebnisse. Psychiat Prax 2007; 34: 26 - 33

9 National Institute for Clinical Excellence. Clinical Practice Guidelines for Violence: The Short-term Management of Disturbed/Violent Behaviour in Psychiatric In-patient Settings and Emergency Departments. February 2005

10 Steinert T, Kallert TW. Medikamentöse Zwangsbehandlung in der Psychiatrie. Psychiat Prax 2006; 33: 160- 169

11 Sailas E, Fenton M. Seclusion and restraint for people with serious mental illnesses (Cochrane Review). The Cochrane Database of Systematic Reviews, 2000, Issue 1. Art. No.: CD001163. DOI: 10.1002/ 14651858.CD001163

12 LeBel J, Stromberg N, Duckworth K, Kerzner J, Goldstein R, Meeks M, Harper G, LaFlair L, Sudders M. Child and Adolescent Inpatient Restraint Reduction: A State Initiative to Promote Strength-Based Care. J Am Acad Child Adolesc Psychiatry 2004; 43: 37-45

13 Smith GM, Davis RH, Bixler EO, Lin HM, Altenor A, Altenor RJ, Hardenstine BD, Kopchick GA. Pennsylvania State Hospital System's Seclusion and Restraint Reduction Program. Psychiatr Serv 2005; 56: 1115 1122

14 Richter D, Needham I. Effekte von mitarbeiterbezogenen Trainingsprogrammen zum Aggressionsmanagement in Einrichtungen der Psychiatrie und Behindertenhilfe - Systematische Literaturübersicht. Psychiat Prax 2007; 34: 7-14

15 Kallert TW, Rymaszewska J, Torres-Gonzáles F. Chapter 15: The Clinical Point of View: Comparing Differences of Legal Regulations Related to Involuntary Admission and Hospital Stay in Twelve European Countries. In: Kallert TW, Torres-González F (eds): Legislation on Coercive Mental Health Care in Europe. Legal Documents and Comparative Assessment of Twelve European Countries. Berlin, Bern, Bruxelles, Frankfurt/M, New York, Oxford, Wien: Peter Lang Europäischer Verlag der Wissenschaften, 2006: 375-400

16 Möller HJ (Hrsg). Therapie psychischer Erkrankungen. 3. Auflage. Stuttgart, New York: Thieme Verlag, 2006

17 Bundesärztekammer. (Muster-)Weiterbildungsordnung und (Muster-) Richtlinien über den Inhalt der Weiterbildung. Stand: Januar bzw. April 2006 